\title{
Genetic association between temperament and sexual precocity indicator traits in Nellore cattle
}

\author{
T. S. Valente • A. C. Sant'Anna • F. Baldi • \\ L. G. Albuquerque • Mateus J. R. Paranhos da Costa
}

Received: 21 May 2014 / Revised: 12 October 2014 / Accepted: 17 November 2014 /Published online: 4 December 2014

(C) Institute of Plant Genetics, Polish Academy of Sciences, Poznan 2014

\begin{abstract}
The aim of this study was to estimate the genetic associations between temperament and sexual precocity indicators in Nellore cattle. Temperament was evaluated at approximately 550 days of age, using three traits: i) movement score (MOV), recording the animals' movement inside the crush; ii) temperament score (TS) for assessing the reactions of animals in a corral pen; and iii) flight speed test (FS), recording the speed (in $\mathrm{m} / \mathrm{s}$ ) at which the animals exit the crush after being weighed. Three sexual precocity indicators were used: i) age at first calving (AFC, in days); ii) occurrence of precocious pregnancy (OPP, as a binary trait); and iii) yearling scrotal circumference $(\mathrm{SC}$, in $\mathrm{cm}$ ). The (co)variance and genetic parameters were estimated by Bayesian Inference via Gibbs sampling, assuming a linear animal model for FS, AFC and SC and a threshold animal model for MOV, TS and OPP in multitrait analyses. The mean posterior heritability estimates for MOV, FS, TS, AFC, OPP and SC were $0.11 \pm$ $0.03,0.27 \pm 0.07,0.16 \pm 0.09,0.09 \pm 0.01,0.44 \pm 0.06$ and $0.43 \pm 0.02$, respectively. The mean posterior correlation estimates were: $0.13 \pm 0.14$ (MOV-AFC), $-0.03 \pm 0.16$ (MOVOPP), $0.08 \pm 0.10$ (MOV-SC), $0.14 \pm 0.11$ (FS-AFC), $-0.19 \pm$ 0.15 (FS-OPP), $-0.07 \pm 0.08$ (FS-SC), $0.09 \pm 0.09$ (TS-AFC),
\end{abstract}

T. S. Valente · A. C. Sant'Anna • L. G. Albuquerque •

M. J. R. P. da Costa $(\varangle)$

Departamento de Zootecnia, Faculdade de Ciências Agrárias e

Veterinárias, Universidade Estadual Paulista,

14.884-900 Jaboticabal, São Paulo, Brazil

e-mail: mpcosta@fcav.unesp.br

T. S. Valente - A. C. Sant'Anna • F. Baldi • M. J. R. P. da Costa Grupo de Estudos e Pesquisas em Etologia e Ecologia Animal, Jaboticabal 14.884-900, São Paulo, Brazil

L. G. Albuquerque $\cdot$ M. J. R. P. da Costa

CNPq, Brasília, Brazil
$-0.03 \pm 0.09$ (TS-OPP) and $-0.28 \pm 0.08$ (TS-SC). These results indicate that all the traits had sufficient genetic variability to respond to selection. The low magnitude of the genetic correlations indicated that selection for improvements in sexual precocity would not change the temperament of Nellore cattle. In order to obtain genetic progress in cattle temperament, direct selection for these traits is recommended.

Keywords Beef cattle · Flight speed · Genetic correlation . Heritability · Reactivity

\section{Introduction}

It is well recognized that there are negative effects of stress on reproductive efficiency in cattle (Von Borell et al. 2007). As temperamental individuals have higher responsiveness to stress (Cafe et al. 2011), it would be expected that they have higher susceptibility to those negative effects. For instance, Burrow et al. (1988) reported that females with better temperament had higher conception rates in artificial insemination (AI) systems, and Cooke et al. (2011) found a direct effect of temperament on pregnancy rate in Nellore cows subjected to fixed-time AI.

Despite evidence of phenotypic correlations, only a few studies have estimated the genetic associations between temperament and reproductive efficiency in cattle, and there has been wide variation in the estimates of the genetic correlations, ranging from 0.00 to 0.55 (Burrow 2001; Phocas et al. 2006; Barrozo et al. 2012). The great difficulty in reaching a consensus from these studies is partly due to the different methods used for assessing cattle temperament, which have included flight speed (Burrow 2001), temperament scores (Barrozo et al. 2012) and docility tests (Phocas et al. 2006). In addition, some 
studies have assessed reproductive performance (fertility and calving ease) and others sexual precocity traits (age at first calving, days to calving, scrotal circumference). In the latter case, we found only one study which assessed the genetic association between a temperament trait (temperament score) and sexual precocity traits in Nellore cattle (Barrozo et al. 2012).

In a previous investigation, we showed that temperament traits would respond to selection in Nellore cattle (Sant'Anna et al. 2013), but this was not enough to recommend their inclusion as selection criteria, as it is necessary to know the genetic association between temperament and other traits which are already used in the breeding programs, such as sexual precocity. Thus, this study was developed in order to estimate the genetic association between temperament and sexual precocity traits in Nellore cattle.

\section{Materials and methods}

This research was approved by the Committee of Ethical Use of Animals from the Faculty of Agricultural and Veterinarian Sciences, São Paulo State University, Jaboticabal-SP, Brazil (Certified n. 007808/11). Data collection was conducted in a Nellore herd belonging to Agropecuária Jacarezinho ${ }^{\circledR}$ Ltda. with two production units located in São Paulo and Bahia states, Brazil. The cattle were kept on tropical pastures, and received mineral supplementation throughout the lasting year. There were two breeding seasons, one from November to January lasting 70 days for multiparous females and 60 days for heifers (females of approximately 24 months of age) and; the other from February to April, also lasting 70 days but involving only young females (between 15 and 17 months of age) in order to identify and select sexually precocious heifers.

Soon after birth, calves were separated by sex and assigned to handling groups. At weaning (approximately 210 days of age), visual scores (conformation, finishing precocity and muscling) and weighing were performed. These records were used in a selection index which was used to select animals for retention in the herd, based on exclusion of $50 \%$ of the males and $10 \%$ of the females. Selected animals were relocated to new handling groups, where they remained until the yearling (approximately 550 days of age). At yearling, a second performance evaluation was conducted including visual scores, weight, scrotal circumference, height, breed characteristics and temperament. Based on this information a new selection index was calculated following the same criteria where $50 \%$ of the males and $10 \%$ of the females were culled. The method of independent culling levels of selection was applied for breed characteristics and temperament, culling animals with the worst grades (Conexão Delta 2011).

In this study, temperament assessments were performed simultaneously to the performance evaluation at yearling, while aiming to minimize interference in the farm handling routine.

Indicators of temperament

Three measures of temperament were used: movement score (MOV), flight speed (FS) and temperament score (TS). The movement score evaluated the animals' movement inside the cattle crush applying the following predefined scores: $1=$ no movement; 2 =little movement, during less than half of the observation time (which was $4 \mathrm{~s}$ ); 3 =frequent movements (during half of the observation time or more), but not vigorous; $4=$ constant and vigorous movements; and $5=$ constant and vigorous movements, animal jumps and raises the limbs off of the ground (adapted from Fordyce et al. 1982).

Flight speed (FS) (Burrow et al. 1988), assessed the speed at which each animal left the cattle crush after weighing. The time taken by each animal to cover a known distance was registered, and the distance varied between 1.6 and $2.0 \mathrm{~m}$, according to the facilities. The information was recorded with an electronic device, comprising two pairs of photoelectric cells, a stopwatch and a processor. From the data for time and distance, flight speed was calculated in meters per second $(\mathrm{m} / \mathrm{s})$. The animals with higher speed were considered the worst temperament.

Temperament score (TS) was used in the Agropecuária Jacarezinho ${ }^{\circledR}$ Ltda (Conexão Delta 2011) breeding program as an independent selection criterion. It gives a score for each animals' reaction after leaving the crush and entering a corral pen as follows: $1=$ the animal walks slowly, allowing close proximity to the observer; $2=$ trots or runs for a few seconds, allowing a moderate proximity to the observer; $4=$ runs during the entire observation time, looking for an escape with constant movement of the tail, and does not allow close or moderate proximity; and $5=$ runs during the entire time of the assessment, jumps against fences and obstacles, and tries to attack the observer. To avoid the tendency of the evaluators concentrating the grades in the intermediary level $(\mathrm{TS}=3)$, it was removed from the scale, thus scoring the animals as $1,2,4$ or 5 .

Sexual precocity traits

For sexual precocity indicator traits, a database with information from animals born between 1990 and 2009 was used. Age at first calving (AFC) is a trait related to the onset of a females' reproductive activity, and it was calculated as the difference in days between the date of the first calving and the date of the dam's birth. The occurrence of precocious pregnancy (OPP) was also used to evaluate the heifers' ability to calve before 30 months of age, and it was recorded as a binary trait, considering " 1 " for the heifers that failed in calving before 30 months of age or "2" when they succeeded in achieving it. 
The data showed that $12 \%$ of the females were considered precocious. Scrotal circumference (SC) was measured (in $\mathrm{cm}$ ) at yearling, in the wider region of the scrotum using a measuring tape.

\section{Statistical analysis}

The weaning weight (WW) was only included in the data analyses as an anchor trait, to minimize the influence of sequential selection and culling at weaning on genetic parameter estimates at the yearling age. Contemporary groups $(\mathrm{CG})$ included the effects of farm and year of birth, sex and management groups at birth and weaning for WW. For temperament traits (MOV, FS and TS) management groups at yearling were also included. For AFC, OPP and SC, the CG included the same effects as for temperament traits excluding sex. For all traits, $C G$ with fewer than five animals were not considered in the analyses. For WW, FS, AFC and SC, the records out of the range according to the mean of the $\mathrm{CG} \pm 3$ standard deviations were excluded from the analyses. The descriptive statistics for temperament and sexual precocity traits are presented in Table 1 and the distribution for MOV, TS and OPP in Table 2. Weaning weight data from 108,386 calves, born from 38,884 dams and 759 bulls, with mean WW of $172.29 \pm$ $26.47 \mathrm{~kg}$ were used.

The (co)variance components and genetic parameters were estimated with Bayesian Inference, using Gibbs sampling in the THRGIBBS1F90 software (Misztal et al. 2002). The multiple-traits analyses included one temperament trait, one sexual precocity trait and WW. Linear animal models were used for WW, FS, AFC and SC and threshold models for MOV, TS and OPP. The Bayesian threshold model was based on the assumption that the levels of the categorical variable were related to an underlying continuous scale containing the fixed and random effects (Van Tassell et al. 1998), this being suitable for genetic analyses of categorical traits.

The model included direct additive genetic and residual effects as random effects, $\mathrm{CG}$ and age of dam at calving, in classes (ranging from 2 to 14 years old) as fixed effects. Only for WW the model included maternal additive genetic and
Table 2 Frequencies for movement score (MOV), temperament score (TS) and occurrence of precocious pregnancy (OPP) for Nellore cattle

\begin{tabular}{llll}
\hline Score & MOV $(N=7415)$ & TS $(N=23,420)$ & OPP $(N=12,756)$ \\
\hline 1 & $14.87 \%$ & $8.84 \%$ & $87.61 \%$ \\
2 & $12.22 \%$ & $84.93 \%$ & $12.38 \%$ \\
3 & $2.51 \%$ & - & - \\
4 & $0.96 \%$ & 5.36 & - \\
5 & $0.01 \%$ & 0.87 & - \\
\hline
\end{tabular}

maternal permanent environment effects as random effects. Age of animal at the time of measurement was included as covariate (with linear and quadratic effects for WW, TS, AFC, $\mathrm{OPP}$ and $\mathrm{SC}$, and linear for the remaining traits). The matrix presentation of the general model used is:

$y=X \beta+Z_{1} a+Z_{2} m+Z_{3} c+e$,

where $\boldsymbol{y}$ is the vector of observations; $\boldsymbol{\beta}$ is the vector of fixed effects; $\boldsymbol{a}$ is the vector of direct additive genetic effects; $\boldsymbol{m}$ is the vector of maternal additive genetic effects; $\boldsymbol{c}$ is the vector of maternal permanent environment effects; and $\boldsymbol{e}$ is the vector of residual effects; and $\boldsymbol{X}, \boldsymbol{Z}_{1}, \boldsymbol{Z}_{2}$ and $\boldsymbol{Z}_{3}$ are incidence matrices relating $\boldsymbol{\beta}, \boldsymbol{a}, \boldsymbol{m}$ and $\boldsymbol{c}$ to $\boldsymbol{y}$. It was assumed that $\mathrm{E}[\mathrm{y}]=X \beta$; $\operatorname{Var}(a)=A \oplus G ; \operatorname{Var}(m)=A \oplus G_{m} ; \operatorname{Var}(c)=I \otimes M P E$ and $\operatorname{Var}(e)=$ $I \otimes R$, where $\boldsymbol{A}$ is the relationship matrix among all animals in the pedigree file, is the direct product, $\boldsymbol{G}$ is the (co)variance matrix of direct additive genetic effects, $\boldsymbol{G}_{\boldsymbol{M}}$ is the (co)variance matrix of maternal additive genetic effects, $\boldsymbol{M P E}$ is the (co)variance matrix of maternal permanent environmental effects, $\boldsymbol{I}$ is the identity matrix and $\boldsymbol{R}$ is the (co)variance matrix of residual effects. The maternal genetic and permanent maternal environment effects were included only for WW, assuming that they were not correlated to residual effect.

The vectors $\boldsymbol{\beta}, \boldsymbol{a}, \boldsymbol{m}$ and $\boldsymbol{c}$ are location parameters from the conditional distribution. A uniform distribution of $\boldsymbol{\beta}$ was assumed a priori, which reflects a vague prior knowledge about this vector. For (co)variances matrices of random effects, inverted Wishart distributions were

Table 1 Number of observations, mean (SD), median, number of CG, bull and dams used for MOV, FS, TS, AFC, OPP and SC

\begin{tabular}{lllllrr}
\hline Traits & Number of observations & Mean (SD) & Median & CG & Number of bulls & Number of dams \\
\hline MOV & 7415 & - & 2 & 349 & 297 & 6359 \\
FS (m/s) & 7402 & $2.26(1.00)$ & - & 350 & 294 & 6351 \\
TS & 23,420 & - & 2 & 741 & 505 & 14,226 \\
AFC (days) & 26,036 & $1070.30(100.25)$ & - & 1,374 & 513 & 17,357 \\
OPP & 12,756 & - & 1 & 1,260 & 387 & 9679 \\
SC (cm) & 34,195 & $26.70(3.10)$ & - & 976 & 661 & 18,066
\end{tabular}

$C G$ contemporary group, $M O V$ movement score, $F S$ flight speed, $T S$ temperament score, $A F C$ age at first calving, $O P P$ occurrence of precocious pregnancy, $S C$ scrotal circumference 
Table 3 Posterior genetic correlation estimates between temperament (MOV, FS and TS) and sexual precocity (AFC, OPP and SC) traits

\begin{tabular}{lrrrr}
\hline Traits & Mean (SD) & Median & \multicolumn{2}{l}{ HPD (95 \%) } \\
\hline MOV-AFC & $0.13(0.14)$ & 0.12 & -0.13 & 0.41 \\
MOV-OPP & $-0.03(0.16)$ & -0.02 & -0.35 & 0.27 \\
MOV-SC & $0.08(0.10)$ & 0.07 & -0.10 & 0.30 \\
FS-AFC & $0.14(0.11)$ & 0.14 & -0.08 & 0.35 \\
FS-OPP & $-0.19(0.15)$ & -0.20 & -0.43 & 0.05 \\
FS-SC & $-0.07(0.08)$ & -0.07 & -0.23 & 0.1 \\
TS-AFC & $0.09(0.09)$ & 0.09 & -0.10 & 0.27 \\
TS-OPP & $-0.03(0.09)$ & -0.03 & -0.20 & 0.17 \\
TS-SC & $-0.28(0.08)$ & -0.29 & -0.41 & -0.11 \\
\hline
\end{tabular}

$M O V$ movement score, $F S$ flight speed, $T S$ temperament score, $A F C$ age at first calving, $O P P$ occurrence of precocious pregnancy, $S C$ scrotal circumference, $H P D$ highest posterior density region

defined as prior distributions. Thus, the distribution of $y$ given the parameters of location and scale was assumed (Van Tassel and Van Vleck 1996):

$y \mid \beta, a, m, c, R \sim M V N\left[X \beta+Z_{1} a+Z_{2} m+Z_{3} c, I_{\mathbf{N}} R\right]$.

On multivariate analyses, chains of 800,000 iterations were generated. The first 20,000 iterations were discarded. For parameter estimates, samples were kept each 100 cycles, generating chains with 7800 cycles. Data convergence was checked through graphical analysis, sampled values versus rounds, and using the criteria proposed by Geweke (1992), Heidelberger and Welch (1983) and Raftery and Lewis (1992) using the R software, with Bayesian Output Analysis (BOA) package from R 2.9.0 software. The pedigree file included 327,309 animals, 1042 bulls and 101,494 dams.

\section{Results}

According to the convergence criteria applied, the number of cycles, burn-in period and the number of Markov chains were sufficient for convergence of the estimated parameters. The mean ( \pm standard deviation) of posterior heritabilities for temperament traits were low for MOV $(0.11 \pm 0.03)$, followed by TS $(0.16 \pm 0.09)$ and FS $(0.27 \pm 0.07)$. The posterior heritability estimates for sexual precocity traits had a low mean value ( \pm standard deviation) for AFC $(0.09 \pm 0.01)$, and high values for OPP $(0.44 \pm 0.06)$ and SC $(0.43 \pm 0.02)$.

The posterior means of the genetic correlation estimates between temperament and sexual precocity traits were all low (Table 3). The highest correlation was between SC and TS $(-0.28)$, indicating that higher scrotal circumference was related to lower temperament score (better temperament). The genetic correlation estimates between $\mathrm{AFC}$ and temperament traits were all low and positive, ranging from 0.09 to 0.14, while for OPP most of the estimated correlations with the temperament traits were close to zero, except with FS (-0.19).

\section{Discussion}

The mean posterior heritability estimates for MOV, FS and TS have already been considered in one of our previous papers (Sant'Anna et al. 2013), and they were within the range found in the literature for European and zebu cattle, and their crosses (Burrow and Corbet 2000; Prayaga et al. 2009; Barrozo et al. 2012; Piovezan et al. 2013). All those results, including the present, indicate that selecting animals for lower values of MOV, FS and TS may result in genetic changes for these traits, and this would be expected to improve cattle temperament.

The magnitude of the posterior heritability estimates for the sexual precocity traits was also close to the values reported in the literature for Nellore cattle (Dias et al. 2004; Eler et al. 2004; Shiotsuki et al. 2009; Boligon and Albuquerque 2011). The estimated heritability for AFC indicates that this trait is greatly influenced by environmental factors to which the females are exposed. Other authors also found low heritability values for AFC (ranging from 0.06 to 0.19), leading then to suggest that part of the genetic variation for this trait cannot be estimated due to the short time allowed for the animals in the breeding season, and to the exclusion of females that did not calve during the analysis, both of which could reduce the heritability estimates (Dias et al. 2004; Forni and Albuquerque 2005; Boligon and Albuquerque 2011). Conversely, the heritability reported in the literature for OPP are higher than ours, ranging from 0.50 to 0.69 (Eler et al. 2002, 2004; Shiotsuki et al. 2009), indicating that this trait can respond satisfactorily to selection. As suggested by Eler et al. (2004), the use of OPP is more efficient than AFC because its evaluation can be made from pregnancy diagnosis (prior to birth) and confirmed after calving, besides the higher values of its heritability.

The $\mathrm{SC}$ is used as an indirect selection criterion for female sexual precocity in many cattle breeding programs, since it is genetically correlated, in the desirable direction, with sexual precocity of females (Forni and Albuquerque 2005; Grossi et al. 2009). Our heritability estimate for SC is within the range reported for this trait (from 0.31 to 0.57 according to Eler et al. 2004; Boligon et al. 2010; Barrozo et al. 2012), and we can confirm the advantages of its use as a way of improving female sexual precocity, since it is moderately heritable, higher than AFC for example, and is very easy to obtain on farm.

In general, the genetic correlation estimates between temperament indicators and $\mathrm{AFC}, \mathrm{OPP}$ and $\mathrm{SC}$ were in the 
favorable direction, indicating that better temperament (lower scores) could be genetically associated with higher sexual precocity. However, considering the low mean values of the estimates and the width of highest posterior density regions (HPD), we are not able to confirm that most of those traits are genetically correlated. These results indicate that there are distinct genetic mechanisms controlling the expression of cattle temperament and the onset of reproductive life.

Reactivity in the crush (here expressed as a movement measure) is one of the most widely used measures of cattle temperament (Grandin 1993; Burrow and Corbet 2000; Kadel et al. 2006; Sant'Anna et al. 2013), and yet no information was found in the scientific literature on its genetic association with sexual precocity. Thus, there is a need to expand studies on the genetic relationships between cattle temperament traits and other traits of economic interest.

The posterior mean genetic correlation estimates of FS with $\mathrm{AFC}$, OPP and $\mathrm{SC}$ were both in the favorable direction, although of low magnitude. These findings corroborate reports by Burrow (2001), who found low genetic associations for FS with pregnancy rate $(0.00)$, days to calving $(0.15)$ and yearling SC (0.22), all obtained from two composite herds (Australian Belmont Red breed). The author concluded that, although favorable, these estimates of the genetic correlations indicate that temperament is an independent trait. The present results indicate that FS can be included in Nellore breeding programs, without affecting negatively the sexual precocity traits, AFC and SC, which are widely used as selection criteria.

Like the other indicators of temperament, TS showed genetic correlations close to zero with OPP and AFC. A higher value of correlation was found between TS and SC (-0.28), however, even in this case would not be expected genetic changes on cattle temperament due to the selection applied for higher SC. The estimates obtained in this study were higher than those described by Barrozo et al. (2012), who reported low genetic correlations for a comparable temperament score with AFC $(0.06 \pm 0.19)$ and $\mathrm{SC}(0.07 \pm 0.07)$ for Nellore cattle. Results discrepant to ours have been reported by Phocas et al. (2006) for Limousin cattle, who found higher genetic correlations between a temperament trait (the docility score adapted from Boivin et al. 1992; Le Neindre et al. 1995) and three indicators of reproductive efficiency (age at puberty, percentage of calving heifers and calving ease), reporting genetic correlation estimates of $-0.32,0.55$ and 0.13 , respectively. Different from us, these authors concluded that, genetically, the more docile females will have greater sexual precocity, fertility and calving ease. It should be highlighted that their study examined Limousin breed, which has been selected for calmer temperament for decades in different parts of the world, such as North American, France and Australia.

Although temperament and reproductive performance may be physiologically related, as suggested by Cooke et al. (2009,
2011), our findings indicate that these traits have a weak genetic association. The present results are in accordance with others which used a similar approach but for growth performance, reporting that cattle temperament was not genetically correlated with WW and ADG (Burrow and Corbet 2000; Burrow 2001; Sant'Anna et al. 2012). In general all these findings lead us to hypothesize that, in extensive conditions, cattle temperament seems to be an independent trait which is not related to overall performance.

\section{Conclusion}

The selection criteria currently applied in Nellore breeding programs for increasing female sexual precocity will not be effective in improving cattle temperament, considering the low values of genetic correlation estimated. Thus, in order to obtain genetic progress in Nellore temperament it is recommended to include these traits as selection criteria in the breeding programs. It could be done using any one of the temperament indicators assessed in this study, since all of them presented sufficient genetic variation to enable a selection response.

Acknowledgments This research was funded by the Fundação de Amparo à Pesquisa do Estado de São Paulo - FAPESP (2009/53609-7) and Coordenação de Aperfeiçoamento de Pessoal de Nível Superior CAPES. The study was part of the master thesis of the lead author, prepared for the Post Graduate Programme in Genetics and Animal Breeding at Universidade Estadual Paulista Júlio de Mesquita Filho (UNESP/São Paulo State University), Jaboticabal Campus.

\section{References}

Barrozo D, Buzanskas ME, Oliveira JA, Munari DP, Neves HHR, Queiroz SA (2012) Genetic parameters and environmental effects on temperament score and reproductive traits of Nellore cattle. Animal 6:36-40

Boivin X, Le Neindre P, Chupin JM, Garel JP, Trillat G (1992) Influence of breed and early management on ease of handling and open-field behaviour of cattle. Appl Anim Sci 20:259-277

Boligon AA, Albuquerque LG (2011) Genetic parameters and relationships of heifer pregnancy and age at first calving with weight gain, yearling and mature weight in Nellore cattle. Livest Sci 141:12-16

Boligon AA, Silva JAV 2nd, Sesana RC, Sesana JC, Junqueira JB, Albuquerque LG (2010) Estimation of genetic parameters for body weights, scrotal circumference, and testicular volume measured at different ages in Nellore cattle. J Anim Sci 88:1215-1219

Burrow HM (2001) Variances and covariances between productive and adaptive traits and temperament in composite breed of tropical beef cattle. Livest Prod Sci 70:213-233

Burrow HM, Corbet NJ (2000) Genetic and environmental factors affecting temperament of zebu and zebu-derived beef cattle grazed at pasture in the tropics. Aust J Agric Res 55:155-162

Burrow HM, Seifert GW, Corbet NJ (1988) A new technique for measuring temperament in cattle. Proc Aust Soc Anim Prod 17:154-157 
Cafe LM, Robinson DL, Ferguson DM, Geesink GH, Greenwood PL (2011) Temperament and hypothalamic-pituitary-adrenal axis function are related and combine to affect growth, efficiency, carcass, and meat quality traits in Brahman steers. Domest Anim Endocrinol 40:230-240

Conexão Delta G (2011) Sumário de Touros: Hereford, Braford e Nelore 2011. GenSys, Brazil. http://www.gensys.com.br. Accessed 20 June 2013

Cooke RF, Arthington JD, Araujo DB, Lamb GC (2009) Effects of acclimation to human interaction on performance, temperament, physiological responses, and pregnancy rates of Brahman-crossbred cows. J Anim Sci 87:4125-4132

Cooke RF, Bohnert DW, Meneghetti M, Losi TC, Vasconcelos JLM (2011) Effects of temperament on pregnancy rates to fixed-timed $\mathrm{AI}$ in Bos indicus beef cows. Livest Sci 142:108-113

Dias LT, El Faro L, Albuquerque LG (2004) Heritability estimates for age at first calving in Nellore cattle. Braz J Anim Sci 33:97-102

Eler JP, Silva JAV 2nd, Ferraz JBS, Dias F, Oliveira HN, Evans JL, Golden BL (2002) Genetic evaluation of the probability of pregnancy at 14 months for Nellore heifers. J Anim Sci 80:951-954

Eler JP, Silva JAV 2nd, Ferraz JBS (2004) Additive genetic relationships between heifer pregnancy and scrotal circumference in Nellore cattle. J Anim Sci 82:2519-2527

Fordyce G, Goddard ME, Seifert GW (1982) The measurement of temperament in cattle and effect of experience and genotype. Proc Aust Anim Prod 14:329-332

Forni S, Albuquerque LG (2005) Estimates of genetics correlations between days to calving and reproductive and weight traits in Nellore cattle. J Anim Sci 83:1511-1515

Geweke J (1992) Evaluating the accuracy of sampling-based approaches to calculating posterior moments. In: Bernardo JM, Berger JO, David AP, Smith AFM (eds) Bayesian statistics. Clarendon Press, Oxford, pp 169-193

Grandin T (1993) Behavioral agitation during handling of cattle is persistent over time. Appl Anim Behav Sci 36:1-9

Grossi DA, Venturini GC, Paz CCP, Bezerra LAF, Lobo RB, Oliveira JA, Munari DP (2009) Genetic associations between age at first calving and heifer body weight and scrotal circumference in Nellore cattle. $\mathrm{J}$ Anim Breed Genet 126:387-393

Heidelberger P, Welch PD (1983) Simulation run length control in the presence of an initial transient. Oper Res 31:1109-1144

Kadel MJ, Johnston DJ, Burrow HM, Graser HU, Ferguson DM (2006) Genetics of flight time and other measures of temperament and their value as selection criteria for improving meat quality traits in tropically adapted breeds of beef cattle. Aust J Agric Res 57: 1029-1035

Le Neindre P, Trillat G, Sapa J, Ménissier E, Bonnet JN, Chupin JM (1995) Individual differences in docility in Limousin cattle. J Anim Sci 73:2249-2253

Misztal I, Tsuruta S, Strabel T, Auvray B, Druet T, Lee DH (2002) BLUPF90 and related programs (BGF90). In: Proceedings of the 7th World Congress on Genetics Applied to Livestock Production, Montpellier, France, August 2002. Communication no. 28-07

Phocas F, Boivin X, Sapa J, Trillat G, Boissy A, Le Neindre P (2006) Genetic correlations between temperament and breeding traits in Limousin heifers. Anim Sci 82:1-7

Piovezan U, Cyrillo JNSG, Paranhos da Costa MJR (2013) Breed and selection line differences in the temperament of beef cattle. Acta Sci 35:207-212

Prayaga KC, Corbet NJ, Johnston DJ, Wolcott ML, Fordyce G, Burrow HM (2009) Genetics of adaptive traits in heifers and their relationship to growth, pubertal and carcass traits in two tropical beef cattle genotypes. Anim Prod Sci 49:413-425

Raftery AE, Lewis SM (1992) How many iterations in the Gibbs sampler? In: Bernardo JM, Berger JO, David AP, Smith AFM (eds) Bayesian statistics. Clarendon Press, Oxford, pp 763-773

Sant'Anna AC, Paranhos da Costa MJR, Baldi F, Rueda PM, Albuquerque LG (2012) Genetic associations between flight speed and growth traits in Nellore cattle. J Anim Sci 90:3427-3432

Sant'Anna AC, Paranhos da Costa MJR, Baldi F, Albuquerque LG (2013) Genetic variability for temperament indicators of Nellore cattle. J Anim Sci 91:3532-3537

Shiotsuki L, Silva JAV 2nd, Albuquerque LG (2009) Genetic associations of heifer pregnancy at 16 months with weaning weight and average daily gain from weaning to yearling in Nellore cattle. Braz J Anim Sci 38:1211-1217

Van Tassell CP, Van Vleck LD (1996) Multiple-trait Gibbs sampler for animal models: Flexible programs for Bayesian and likelihoodbased (co)variance component inference. J Anim Sci 74:2586-2597

Van Tassell CP, Van Vleck LD, Gregory KE (1998) Bayesian analysis of twinning and ovulation rates using a multiple trait threshold model and Gibbs sampling. J Anim Sci 76:2048-2061

Von Borell E, Dobson H, Prunier A (2007) A stress behavior and reproductive performance in female cattle and pigs. Horm Behav $52: 130-138$ 